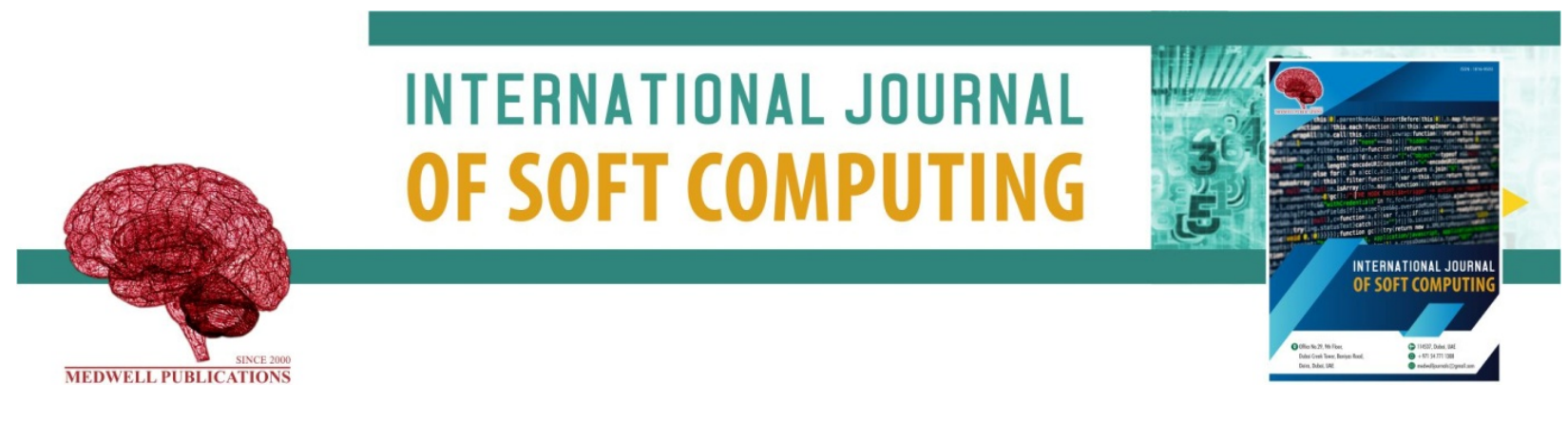

\title{
The Online Social Networks Analysis: State of the Art
}

Asmae El Kassiri and Fatima-Zahra Belouadha

Engineers Mohoammadia School, Mohammed V University, Agdal, Rabat, Morocco

Key words: OSN, SNA, social mining, community detection, link prediction, similarity analysis, recommendation, influence, trust, e-reputation, opinion mining, experts detection, privacy and security on OSN

\section{Corresponding Author:}

Asmae El Kassiri

Engineers Mohoammadia School, Mohammed V University, Agdal, Rabat, Morocco

Page No.: 33-43

Volume: 14, Issue 2, 2019

ISSN: 1816-9503

International Journal of Soft Computing

Copy Right: Medwell Publications
Abstract: Nowadays, the Online Social Networks OSN emergence has expanded the scope of these needs. In fact, besides the direct links between users, the OSN have various important information about user profiles, tastes and interactions or activities. This information richness has been exploited in the literature in particular social mining techniques to answer new analytical purposes such as influence analysis, trust analysis, opinion mining, recommendation, e-reputation, protection of privacy and detection of experts.

\section{INTRODUCTION}

The OSN (Online Social Networks) are the actual communication tool, providing to their members the possibility to express their tastes, interests and activities and to establish relationships. They store huge amount of information that can be analysed in multiple contexts for varied ends.

The OSN analysis combine the structural Social Network Analysis (SNA) to the social mining technics for nine analysis objectives: similarity computing, influence propagation, opinion mining, expert detection, trust analysis, recommendation, privacy, community detection and link prediction.

The aim of this study is double: presenting the different OSN analysis and capturing the indicators used by according algorithms synthetizing. Therefore, for each analysis cited above, the authors have studied multiple works dedicated to identify the objectives, approaches and metrics proposed to compute the need.
This study is organized in eleven sections. The nine next sections corresponds to the analysis applied to OSN and its objectives. The perspective of this work and a conclusion are the subject of the 11th section.

The similarity analysis: Formerly, the similarity concept was associated to a structural similarity based on network topology and neighboring notion. Then, the similarity has evolved to a semantic similarity exploiting the shared contents on social media to evaluate the similitude between profiles or interests. The two notions similarity can be combined to speak about a structural-semanticsimilarity. The proposed approaches are based on three principles.

The neighboring notion (Newman, 2001; Ravasz et al., 2002; Zhou et al., 2009; Leicht et al., 2005; Adamic and Adar, 2003). The hypothesis is that two directly connected persons are similar. It's based on analyzing only the links on the network and it's entitled structural similarity. 
The profiles and interests (O'Donovan and Smyth, 2005; Golbeck, 2009; Bhattacharyya et al., 2011; Akcora et al., 2013; Crandall et al., 2008; Huang and Yang, 2012; Davoodi et al., 2012; Anderson et al., 2012): two users with similar profiles and similar interests are similar. To compute it, geodesics and correlations measures, like Person correlation (Resnick et al., 1994; Konstan et al., 1997) and Spearman correlation (Herlocker et al., 1999) have proven their efficiency. This similarity is entitled semantic similarity.

The hybrid approach (Zhou et al., 2009, 2010; Cruz et al., 2012; Xia and Bu, 2012; Aiello et al., 2012) introduce the semantic similarity to compute structural similarity. It's entitled the structural-semantic similarity.

\section{MATERIALS AND METHODS}

The influence analysis: The influence analysis objectives are identifying trends and popular topics and maximizing/minimizing the influence in an OSN. The influence maximization is possible thanks to the influent nodes able to diffuse to other nodes. The minimization is made by cutting links between the influent nodes and the other nodes (Liu et al., 2010; Guille et al., 2013; Jiang et al., 2014).

Users activities Saito et al., 2008; Anagnostopoulos et al., 2008; Yang and Counts, 2010; Yang and Leskovec 2010; Bakshy et al., 2012; Guille et al., 2013): Using the number of friends who became active (infected or influenced) after an action (Share, comment, like, dislike, etc.). An active friend is a friend who reacts to an action. The reaction can be a share, comment, like, dislike, etc.

Person popularity (Cha et al., 2010; Lagnier et al., 2012): Using the subscribers of his profile page, his publications and his identifications by other users.

Similarity (Crandall et al., 2008): The similarity is a factor of influence.

Structural measures: Kimura and Saito (2006), Chen et al. (2009), Newman (2010), Kim and Yoneki (2012), Mochalova and Nanopoulos (2013), Easley and Kleinberg (2010) and Hinz et al. (2011) using the structural information like centrality and the hubs and switches notions.

Topic popularity: Cha et al. (2009), Asur et al. (2011), Shamma et al. (2011), Alsumait et al. (2008), Cataldi et al. (2010), Guille et al. (2013) and Schubert et al. (2014) using the frequencies of interactions (visits, shares, comments, subscriptions, evaluations, etc.).
Sentiment analysis: Since 2000, the opinion mining (sentiment analysis) became an active research domain. It's founded on statistic and linguistic technics to estimate persons opinions, sentiments, evaluations, attitudes and emotions for entities and its characteristics and attributes (e.g., organizations, products, services, topics, etc.). The opinion mining is studied in multiple researches for different applications like marketing, recommendation, social and political analysis (Liu, 2012, 2015).

The approaches are based on two steps: First, extracting subjective data to identify sentiments and opinions, second, classifying it into positive and negative sentiments (Liu, 2012; Hu and Liu, 2004). The indicators used for the sentiment analysis from OSN are the evaluations (like and dislike).

Expert detection: An expert is a person having a high-level knowledge and skills to perform a domain task. Detecting experts in an OSN aims giving an experts list having high-levels skills able to respond to an organization or a community request. It's mainly explored for the scientific researches to ensure scientific cooperation and recruitments or human resources management to cover human resources needs (Zhang et al., 2007; Karimzadehgan et al., 2009; Lappas et al., 2009; Lappas et al., 2011; Davoodi et al., $2012,2013)$. It can be applied to different networks types like the scientific social networks centred researchers (e.g., ResearchGate) or centred articles like digital librairies (e.g., IEEE Xplore, ACM, CiteSeerx, etc.), the professional social networks (e.g., LinkedIn, Viadeo, etc.), the private enterprise social networks and Questions/Responses sites (e.g., Yahoo!Answers, Quora, Stackoverflow, Comment ca marche, etc.). The objective is identifying experts by estimating their levels skills in a domain and detecting relationships between experts if an experts team is needed to cooperate in the same project. As result, the indicators required for the experts detection are the user's skills and their professional relationships.

Trust analysis: The trust is a bilateral relationship (interpreting a confident feeling). It's asymmetric (not reciprocal), non-transitive and spreadable (nearest people's trend to develop crossed trust networks). A panoply of scientific researchers have analyzed the trust in the OSN in many domains like the security and privacy, the marketing, the recommendation and the clustering (Abdul-Rahman and Hailes, 2000; Dimitrakos, 2003).

The trust analysis exploits the explicit trust declared in OSN. A category of approaches considers the similarity 
(Ziegler and Lausen, 2004; Ziegler and Golbeck, 2007), the strength of links based on the frequent interactions, the absence of conflicts between two users (Gilbert and Karahalios, 2009; Xiang et al., 2010) and the influence (Guha et al., 2004; Ziegler and Lausen, 2005; Golbeck and Hendler, 2006; Adali et al., 2010) as indicators of an implicit trust. Another category exploits the graph theory technics and the trust propagation property to elaborate propagation rules and to infer trust links between not connected neighbors in a trust network (inferred trust) (Guha et al., 2004; Hasan et al., 2009; Chakrabortya and Karform, 2012). Another category reuses the popularity and fidelity notions to compute reputation and trust scores of neighbors to predict malicious nodes (predict good and bad reputation), a malicious node is supposed having good trust scores from malicious users. In other approaches, the bad reputation is deduced from the interactions historic (e.g., a passive account becoming suddenly active is likely pirated by a malicious user) (Massa et al., 2005; Caverlee et al., 2008; Nepal et al., 2011).

\section{RESULTS AND DISCUSSION}

Privacy and security analysis: On OSN, users trend sharing private information online causing protection problems. This information is mainly targeted to a specific audience category of each user. Otherwise, the social media managers impose to their users a usage policy to have their data exploitation rights. Furthermore, the OSN must protect their users accounts against any attack form and ensure their sensitive data protection (Singh et al., 2014).

This field objective is eliminating the personal data divulgation risks, authenticating shared contents on social media and detecting the web spams and attacks. In other words, the aims are to control contents visibility on OSN to protect privacy against any espionage risk and to identify non-authentic contents and malicious users to protect the author users. The approaches of privacy and security on OSN propose different demarches according the targeted objective. To ensure the contents visibility control, there are three demarches kinds:

Using visibility levels: Exploiting confidentiality parameters defined by users on their profiles elements and shared contents to prevent divulgating personal information to non-concerning users (Barbian, 2011).

Using a trust measure: Applying trust learning algorithms estimate trust and prevent divulgating personal information to distrusted users (Sherchan et al., 2010; Golbeck et al., 2003).
Using the online reputation: Exploiting explicit or implicit information about online reputation to identify malicious users and neutralize them by social media or reveal them to users to protect themselves (Caverlee et al., 2008; Levien, 2004). To detect attacks and web spams, three steps were identified:

Detecting undesirable elements (spams): An undesirable element can be a shared content (e.g., post, image, video, etc.). To detect spams, algorithms exploit the hypothesis that a spam is an ignored element (e.g., no positive evaluations, no comments, no visits, no private messages with his owner, etc.), able to propagate very rapidly in a brief period. Then, identifying spams is based on user's interactions analysis concerning a shared content and on its sharing intensity on the OSN (Kincaid, 2016; Cao and Caverlee, 2015; Zhenga et al., 2015).

Identifying spammers: It is based on the infidelity notion. The infidelity consists on the fact that a spammer or a malicious user has many contacts, many temporary accounts and a lot of shared contents but little subscribers. Because, a spammer aim propagating his contents to the maximum users and per statistics, a spammer share per day approximatively three times more than normal user but his actualities fil has little interests (Gyongyi et al., 2004; Cao and Caverlee, 2015).

Detecting pirated accounts: It is based on the hypothesis that a pirated account is an account becoming suddenly more active (e.g., more shares, more contacts, more social page's subscriptions, etc.) and less trusted by the OSN users (e.g., unsubscribing from his contents and containers, deleting from contacts list, reporting, etc.) (Gao et al., 2012; Zhenga et al., 2015).

Community detection: The community notion in traditional social networks was associated to a nodes group with more important internal links than external links. Then in OSN, this notion was extended to users group having the same interests and interact more frequently together than with external users (Nguyen et al., 2011). The objective of the community detection is regrouping nodes having the same semantic context (having a minimal semantic/structural similarity) in the same community. The process aims identifying persons having common characteristics to deduce trust relationships or possible links and respond to other objectives like recommendation. There are five approaches.

Reusing and adapting clustering technics by homogeneity or modularity maximization: It considers a social graph enriched by user's attributes 
(Social-Attribute Networks). The process begins with initial clusters (each cluster having a unique node) and then, calculating iteratively the homogeneity/modularity between each clusters pair and regrouping the nearest clusters (Meftahi and Saidi, 2013; Arab and Afsharchi, 2012; Dang and Viennet, 2012).

Reusing and adapting clustering by partitioning technics: There is two demarches. The first one aims partitioning the OSN into $\mathrm{K}$ dense groups the most similar users. It adapts an iterative process partitioning at each step the groups obtained in the precedent step to maximize the internal similarity and minimize the external one. The second demarche principle is partitioning the OSN into groups with the more similar K persons. It's based on an iterative process enriching at each step the minimal groups candidates until obtaining groups with the more similar $\mathrm{K}$ persons. The two demarches exploit the semantic similarity (Zhou et al., 2009, 2010; Davoodi et al., 2012; Dong et al., 2011).

Using the clustering by oriented interactions regrouping technics: It regroups the most interacting users between them. There are two demarches. The first one operates by transforming the social graph $G$ into a Line Graph G', the G links become the G' nodes and the G nodes become the $G^{\prime}$ links and then regrouping in $G^{\prime}$ the similar nodes (relationships) and finally replacing the each according relationship by its both members allowing a user to belong to different groups. It's essentially used to detect fuzzy communities. The second demarche is founded on rules defined to guide community detection. The process begins by identifying a semantic relationship between two users and then, per the defined rules, verifying if the relationship imposes their regrouping in the same community or not (Palla et al., 2005; Evans and Lambiotte, 2009; Tang and Liu, 2008; Cai et al., 2005; Yoshida, 2012; Sun et al., 2009; Qi et al., 2012; Zhou and Liu, 2013).

Using the clustering by oriented contents regrouping technics: It regroups the OSN users based their shared contents. The hypothesis is that two similar users share similar contents. The process calculates the shared contents similarity to regroup the contents the more similar in the same group and then identifies for each user his groups (a user belongs of each group of his shared contents) (Huang and Yang, 2012).

Using the clustering by oriented trust regrouping technics: It exploits the trust notion to regroup the OSN users trusting each other in the same community. The process estimates the trust inter-users to identify communities (Adali et al., 2010).

Link prediction: The link prediction aims analyzing the topological network actual state to predict the eventual connections appearance/disappearance in the future. While the semantic prediction links explores the OSN using semantic web technics for the same objective. The objective is suggesting new connections between OSN users recommending contents and characterizing the relationships (Gong et al., 2012). There are many approaches.

Exploiting the sociology notions: Per sociologies those notions encourage the relationships establishment between users. The first notion is the hemophilia giving the hypothesis that similar users assemble. This hypothesis exploit the similarity between profiles and interests (semantic similarity), the structural similarity and the structural-semantic similarity to recommend new links. The second notion is the social balance designed also by the triadic closure, giving the hypothesis that friend of my friend are also my friends. This hypothesis allows recommending to a user the contacts of his direct contacts (Akcora et al., 2013; Eagle, 2008; Crandalla et al., 2010; Weng et al., 2013; Gong et al., 2012, 2014).

Exploiting contents or interest's semantic similarity: Calculating similarities between users or shared contents to infer new links between contents or between users and contents. It's identifying users having similar interests to recommend them their similar preferred contents or identifying similar contents to recommend, per example, inter-contents co-citations (Popescul and Ungar, 2003; Parimi and Caragea, 2011; Rawashdeh et al., 2013; Schifanella et al., 2010; Aiello et al., 2012; Chelmis and Prasanna, 2013).

Analyzing the interactions nature and frequencies: Identifying evaluations interactions and calculating those interactions frequencies from two different users concerning the same objects and the trust core between them to deduce eventual conflicts based on their agreements/disagreements score and to weight the relationship based on their agreements/disagreements score and their trust score. If agreements $>$ disagreements and the trust $>0$ then, the relationship is strong (Leroy et al., 2010; Xiang et al., 2010; Leskovec et al., 2010; Symeonidis and Mantas, 2013; Javari and Jalili, 2014; Cai et al., 2012; Tang et al., 2011, 2012). Table 1 resume all the cited indicators. 
Int. J. Soft Comput., 14 (2): 33-43, 2019

Table 1: Indicators synthesis

\begin{tabular}{ll}
\hline Indicators & Used information \\
\hline Structural similarity & Contacts \\
Semantic similarity & Profile property (age, gender, sexual orientation, religion, political orientation, city, country, etc.) \\
Structural-semantic similarity & Container (social group, social page user home, channel video, forum, blog, mailing list, \\
Predominant sentiment & scientific journal, digital library, questions/ responses site, etc.) \\
trust/popularity & Tag \\
Interaction degree & Topic \\
Implicit trust & Interaction (share, citation,cco-citation, evaluation inferred trust (like, dislike), comment, response, visit, \\
Explicit trust/Inferred trust & subscribe, unsubscribe, trust, distrust, recommend, manage privileges, etc.) \\
Influence/conflic & Person \\
Agreement & Declared trust \\
Disagreement & Security profile \\
Fidelity & Security group \\
Reputation & Privilege (see, modify, share, comment, modify privileges, etc.) \\
Relationship weight/Spam/spammer & Skill \\
Visibility level & Project \\
Pirated account & Content (scientific paper, post, image, video, private message, etc.) \\
Indirect relationship expertise & \\
professional link/(collaboration, & \\
coauthoring/co-citation, etc.) & \\
\hline
\end{tabular}

\section{CONCLUSION}

In this study, the OSN analysis identification process studied a documents corpus composed by 149 documents repartitioned on the different analysis.

From the study presented in this study, it's possible to note that the different approaches, studied in this context, use approximatively the same indicators.

Let's remember that this study final objective is constructing an application ontology dedicated to the OSN analysis. The carried out study in this study permit identifying indicators and required basic data to compute it. The aim is to have the precomputed indicators ready for the use in the different analysis approaches to reduce the analysis data preparation time. The future works will be dedicated to the application ontology and its benefits to reduce analysis time.

\section{RECOMMENDATION}

The recommendation systems aim proposing individual recommendations adapted to the users' needs and preferences. Multiple researches works has analyzed recommendation for different domains like marketing, politics and social life (O'Donovan and Smyth, 2005). Two recommendation approaches classes: based-contents recommendation and based-collaborative filtering recommendation (Ricci et al., 2011). The first-class studies user preferences in the past to propose him similar elements. It's based on a process of three steps (Lops et al., 2011):

Contents analysis: Extracting subjective information (positive and negative sentiments) from unstructured contents (e.g., posts and messages raw texts) or from online evaluation interactions (like/dislike).
Profile learning: Identifying positive and negative sentiments scores expressed by a user for an object.

Components filtering: Evaluating similarities between old interests and the new objects to recommend (Sellami et al., 2012). The second class aims proposing recommendations to a user per his contacts interests. It uses two methods (O'Donovan and Smyth, 2005):

Exploiting similarities between users: Analyzing first the old interests of each user to detect similarities between users and then propose to users the recent interests of their more similar contacts (Sulieman et al., 2013; Kadima and Malek, 2013).

Exploiting the declared trust between users to resolve the Cold Start problem (a case of a novel user when it's impossible to analyze his interactions historic). This method can be used in OSN permitting users declaring explicitly trusts scores between users. The idea is to propose to a user his trusted contacts interests (O'Donovan and Smyth, 2005; Golbeck and Hendler, 2005; Haydar, 2014; Xie, 2014).

\section{REFERENCES}

Abdul-Rehman, A. and S. Hailes, 2000. Supporting trust in virtual communities. Proceedings of the $33 \mathrm{rd}$ Hawaii International Conference on System Sciences, January 4-7, 2000, Maui, Hawaii, pp: 6007.

Adali, S., R. Escriva, M.K. Goldberg, M. Hayvanovych and M. Magdon-Ismail et al., 2010. Measuring behavioral trust in social networks. Proceedings of the IEEE International Conference on Intelligence and Security Informatics, May 23-26, 2010, Vancouver, BC., Canada, pp: 150-152.

Adamic, L.A. and E. Adar, 2003. Friends and neighbors on the web. Social Netw., 25: 211-230. 
Aiello, L.M., A. Barrat, R. Schifanella, C. Cattuto and B. Markines et al., 2012. Friendship prediction and homophily in social media. ACM. Trans. Web, 6: $1-33$.

Akcora, C.G., B. Carminati and E. Ferrari, 2013. User similarities on social networks. Soc. Netw. Anal. Min., 3: 475-495.

AlSumait, L., D. Barbara and C. Domeniconi, 2008. On-line LDA: Adaptive topic models for mining text streams with applications to topic detection and tracking. Proceedings of the 8th IEEE International Conference on Data Mining (ICDM'08), December 15-19, 2008, IEEE, Pisa, Italy, ISBN:978-0-76953502-9, pp: 3-12.

Anagnostopoulos, A., R. Kumar and M. Mahdian, 2008. Influence and correlation in social networks. Proceedings of the 14th ACM SIGKDD International Conference on Knowledge Discovery and Data Mining (KDD '08), August 24-27, 2008, ACM, Las Vegas, Nevada, USA., ISBN:978-1-60558-193-4, pp: $7-15$.

Anderson, A., D. Huttenlocher, J. Kleinberg and J. Leskovec, 2012. Effects of user similarity in social media. Proceedings of the 5th ACM International Conference on Web Search and Data Mining (WSDM '12), February 08-12, 2012, ACM, Seattle, Washington, USA., ISBN:978-1-4503-0747-5, pp: 703-712.

Arab, M. and M. Afsharchi, 2012. A modularity maximization algorithm for community detection in social networks with low time complexity. Proceedings of the 2012 IEEE/WIC/ACM International Joint Conferences on Web Intelligence and Intelligent Agent Technology (WI-IAT '12) Vol. 1, December 4-7, 2012, IEEE Computer Society, Washington, DC, USA., ISBN:978-0-7695-4880-7, pp: 480-487.

Asur, S., B.A. Huberman, G. Szabo and C. Wang, 2011. Trends in social media: Persistence and decay. Proceedings of the 5th International AAAI Conference on Weblogs and Social Media, July 17-21, 2011, The AAAI Press, Barcelona, Catalonia, Spain, pp: 334-337.

Avesani, P., P. Massa and R. Tiella, 2005. A trust-enhanced recommender system application: Moleskiing. Proceedings of the 2005 ACM International Symposium on Applied Computing (SAC '05), March 13-17, 2005, ACM, Santa Fe, New Mexico, ISBN:1-58113-964-0, pp: 1589-1593.

Bakshy, E., I. Rosenn, C. Marlow and L. Adamic, 2012. The role of social networks in information diffusion. Proceedings of the 21st international conference on World Wide Web, April 16-20, 2012, ACM, New York, USA., ISBN: 978-1-4503-1229-5, pp: 519-528.
Barbian, G., 2011. Trust centrality in online social networks. Proceedings of the 2011 European Conference on Intelligence and Security Informatics, September 12-14, 2011, IEEE, Athens, Greece, ISBN:978-1-4577-1464-1, pp: 372-377.

Bhattacharyya, P., A. Garg and S.F. Wu, 2011. Analysis of user keyword similarity in online social networks. Soc. Netw. Anal. Min., 1: 143-158.

Cai, D., Z. Shao, X. He, X. Yan and J. Han, 2005. Mining hidden community in heterogeneous social networks. Proceedings of the 3rd International Workshop on Link Discovery (LinkKDD '05), August 21-25, 2005, ACM, Chicago, Illinois, ISBN:1-59593-215-1, pp: 58-65.

Cai, X., M. Bain, A. Krzywicki, W. Wobcke and Y.S. Kim et al., 2012. Reciprocal and heterogeneous link prediction in social networks. Proceedings of the 16th Pacific-Asia Conference on Knowledge Discovery and Data Mining (PAKDD 2012), May 29-June 1, 2012, Springer, Berlin, Germany, ISBN:978-3642-30219-0, pp: 193-204.

Cao, C. and J. Caverlee, 2015. Detecting Spam URLs in Social Media via Behavioral Analysis. In: Advances in Information Retrieval, Hanbury, A., G. Kazai, A. Rauber and N. Fuhr (Eds.). Springer, Vienna, Austria, ISBN:978-3-319-16353-6, pp: 703-714.

Cataldi, M., L.D. Caro and C. Schifanella, 2010. Emerging topic detection on Twitter based on temporal and social terms evaluation. Proceedings of the 10th ACM International Workshop on Multimedia Data Mining. July 25-25, 2010, ACM, New York, USA., ISBN:978-1-4503-0220-3, pp:4-10.

Caverlee, J., L. Liu and S. Webb, 2008. Socialtrust: Tamper-resilient trust establishment in online communities. Proceedings of the 8th ACM/IEEE-CS Joint Conference on Digital libraries, June 16-20, 2008, ACM, Pittsburgh, Pennsylvania, USA., ISBN:978-1-59593-998-2, pp: 104-114.

Cha, M., A. Mislove and K.P. Gummadi, 2009. A measurement-driven analysis of information propagation in the flickr social network. Proceedings of the 18th International Conference on World Wide Web (WWW 09), April 20-24, 2009, ACM, Madrid, Spain, ISBN:978-1-60558-487-4, pp: 721-730.

Cha, M., H. Haddadi, F. Benevenuto and P.K. Gummadi, 2010. Measuring user influence in Twitter: The million follower fallacy. Proceedings of the 4th International AAAI Conference on Weblogs and Social Media, May 23-26, 2010, George Washington University, Washington, D.C., USA., pp: 10-17.

Chakraborty, P.S. and S. Karform, 2012. Designing trust propagation algorithms based on simple multiplicative strategy for social networks. Procedia Technol., 6: 534-539. 
Chelmis, C. and V.K. Prasanna, 2013. Social link prediction in online social tagging systems. ACM. Trans. Inf. Syst., 31: 1-27.

Chen, W., Y. Wang and S. Yang, 2009. Efficient influence maximization in social networks. Proceedings of the 15th ACM SIGKDD International Conference on Knowledge Discovery and Data Mining, June 28-July 01, 2009, ACM, New York, USA., ISBN: 978-1-60558-495-9, pp: 199-208.

Crandall, D., D. Cosley, D. Huttenlocher, J. Kleinberg and S. Suri, 2008. Feedback effects between similarity and social influence in online communities. Proceedings of the 14th ACM SIGKDD International Conference on Knowledge Discovery and Data Mining (KDD '08), August 24-27, 2008, ACM, Las Vegas, Nevada, USA., ISBN:978-1-60558-193-4, pp: $160-168$.

Crandall, D.J., L. Backstrom, D. Cosley, S. Suri and D. Huttenlocher et al., 2010. Inferring social ties from geographic coincidences. Proc. Nat. Acad. Sci. USA., 107: 22436-22441.

Dang, T.A. and E. Viennet, 2012. Community detection based on structural and attribute similarities. Proceedings of the 6th International Conference on Digital Society (ICDS), January 30-February 04, 2012, Valencia, Spain, ISBN:978-1-61208-176-2, pp: $7-12$.

Davoodi, E., K. Kianmehr and M. Afsharchi, 2013. A semantic social network-based expert recommender system. Appl. Intell., 39: 1-13.

Davoodi, E., M. Afsharchi and K. Kianmehr, 2012. A social network-based approach to expert recommendation system. Proceedings of the International Conference on Hybrid Artificial Intelligence Systems, March 28-30, 2012, Springer, Berlin, Germany, ISBN:978-3-642-28941-5, pp: 91-102.

Dimitrakos, T., 2002. A service-oriented trust management framework. Proceedings of the International Workshop on Deception, Fraud and Trust in Agent Societies, July 15, 2002, Springer, Berlin, Germany, ISBN:978-3-540-00988-7, pp: 53-72.

Dong, W., C. Moses and K. Li, 2011. Efficient k-nearest neighbor graph construction for generic similarity measures. Proceedings of the 20th International Conference on World Wide Web (WWW '11), March 28-April 01, 2011, ACM, Hyderabad, India, ISBN:978-1-4503-0632-4, pp: 577-586.
Eagle, N., A.S. Pentland and D. Lazer, 2008. Mobile Phone Data for Inferring Social Network Structure. In: Social Computing, Behavioral Modeling and Prediction, Liu, H., J.J. Salerno and M.J. Young (Eds.). Springer, Boston, Massachusetts, USA., ISBN:978-0-387-77671-2, pp: 79-88.

Easley, D. and J. Kleinberg, 2010. Networks, Crowds and Markets: Reasoning About a Highly Connected World. Cambridge University Press, Cambridge, UK., ISBN:978-0-521-19533-1, Pages: 728.

Evans, T.S. and R. Lambiotte, 2009. Line graphs, link partitions and overlapping communities. Phys. Rev. E., 80: 1-15.

Gao, H., Y. Chen, K. Lee, D. Palsetia and A.N. Choudhary, 2012. Towards online spam filtering in social networks. Proceedings of the 19th Annual International Symposium on Network \& Distributed System Security (NDSS-2012), February 05-08, 2012, San Diego, California, USA., pp: 1-16.

Gilbert, E. and K. Karahalios, 2009. Predicting tie strength with social media. Proceedings of the SIGCHI International Conference on Human Factors in Computing Systems (CHI '09), April 04-09, 2009, ACM Boston, Massachusetts, USA., ISBN:978-160558-246-7, pp: 211-220.

Golbeck, J. and J. Hendler, 2006. Inferring binary trust relationships in web-based social networks. ACM. Trans. Internet Technol., 6: 497-529.

Golbeck, J., 2009. Trust and nuanced profile similarity in online social networks. ACM Trans. Web, Vol. 3. $10.1145 / 1594173.1594174$

Golbeck, J., B. Parsia and J. Hendler, 2003. Trust networks on the semantic web. Proceedings of the 7th International Workshop on Cooperative Information Agents, August 27-29, 2003, Springer, Berlin, Germany, ISBN:978-3-540-40798-0, pp: 238-249.

Golbeck, J.A., 2005. Computing and applying trust in web-based social networks. Ph.D Thesis, University of Maryland Libraries, College Park, Maryland.

Gong, N.Z., A. Talwalkar, L. Mackey, L. Huang and E.C.R. Shin et al., 2012. Jointly predicting links and inferring attributes using a Social-Attribute Network (SAN). Proceedings of the 6th International Workshop on SNA-KDD (SNA-KDD'12), August 12, 2012, Beijing, China, ACM, New York, USA., ISBN:978-1-4503-1544-9, pp: 1-9.

Gong, N.Z., A. Talwalkar, L. Mackey, L. Huang and E.C.R. Shin et al., 2014. Joint link prediction and attribute inference using a social-attribute network. ACM. Trans. Intell. Syst. Technol., 5: 1-20.

Guha, R., R. Kumar, P. Raghavan and A. Tomkins, 2004. Propagation of trust and distrust. Proceedings of the 13th International Conference on World Wide Web (WWW'04), May 17-20, 2004, ACM, New York, USA., ISBN:1-58113-844-X, pp: 403-412. 
Guille, A., H. Hacid, C. Favre and D.A. Zighed, 2013. Information diffusion in online social networks: A survey. ACM. Sigmod Rec., 42: 17-28.

Gyongyi, Z., H. Garcia-Molina and J. Pedersen, 2004. Combating web spam with trustrank. Proceedings of the 13th International Conference on Very Large Data Bases (VLDB '04) Vol. 30, August 31-September 03, 2004, ACM, Toronto, Canada, ISBN:0-12-088469-0, pp: 576-587.

Hasan, O., L. Brunie and J.M. Pierson, 2009. Evaluation of the iterative multiplication strategy for trust propagation in pervasive environments. Proceedings of the 2009 International Conference on Pervasive Services (ICPS '09), July 13-17, 2009, ACM, London, UK., ISBN:978-1-60558-644-1, pp: 49-54.

Herlocker, J.L., J.A. Konstan, A. Borchers and J. Riedl, 1999. An algorithmic framework for performing collaborative filtering. Proceedings of the 22nd Annual International ACM SIGIR Conference on Research and Development in Information Retrieval, August 15-19, 1999, Berkeley, California, pp: 230-237.

Hinz, O., B. Skiera, C. Barrot and J.U. Becker, 2011. Seeding strategies for viral marketing: An empirical comparison. J. Marketing, 75: 55-71.

$\mathrm{Hu}, \mathrm{M}$. and B. Liu, 2004. Mining and summarizing customer reviews. Proceedings of the 10th ACM SIGKDD International Conference on Knowledge Discovery and Data, August 22-25, 2004, ACM Press, Washington, USA., pp: 168-177.

Huang, H.H. and H.C. Yang, 2012. Semantic clustering-based community detection in an evolving social network. Proceedings of the 2012 6th International Conference on Genetic and Evolutionary Computing, August 25-28, 2012, IEEE, Kitakushu, Japan, ISBN:978-1-4673-2138-9, pp: 91-94.

Javari, A. and M. Jalili, 2014. Cluster-based collaborative filtering for sign prediction in social networks with positive and negative links. ACM. Trans. Intell. Syst. Technol., 5: 1-19.

Jiang, C., Y. Chen and K.R. Liu, 2014. Evolutionary dynamics of information diffusion over social networks. IEEE. Trans. Signal Proc., 62: 4573-4586.

Kadima, H. and M. Malek, 2013. Toward ontology-based personalization of a recommender system in social network. Intl. J. Comput. Inf. Syst. Ind. Manage. Appl., 5: 499-508.

Karimzadehgan, M., R.W. White and M. Richardson, 2009. Enhancing expert finding using organizational hierarchies. Proceedings of the 13th European Conference on Information Retrieval, April 6-9, 2009 , Springer, Berlin, Germany, ISBN:978-3-642-00957-0, pp: 177-188.
Kim, H. and E. Yoneki, 2012. Influential neighbours selection for information diffusion in online social networks. Proceedings of the 2012 21st International Conference on Computer Communications and Networks (ICCCN), July 30-August 2, 2012, IEEE, Munich, Germany, ISBN:978-1-4673-1543-2, pp: 1-7.

Kimura, M. and K. Saito, 2006. Tractable models for information diffusion in social networks. Proceedings of the 10th European Conference on Principles of Data Mining and Knowledge Discovery, September 18-22, 2006, Springer, Berlin, Germany, ISBN:978-3-540-45374-1, pp: 259-271.

Kincaid, J., 2010. EdgeRank: The secret sauce that makes facebooks news feed tick. TechCrunch, Bay Area, USA. https://techcrunch.com/2010/04/22/facebookedgerank/

Konstan, J.A., B.N. Miller, D. Maltz, J.L. Herlocker and L.R. Gordon et al., 1997. Group Lens: Applying collaborative filtering to Usenet news. Commun. ACM., 40: 77-87.

Lappas, T., K. Liu and E. Terzi, 2009. Finding a team of experts in social networks. Proceedings of the 15th ACM SIGKDD International Conference on Knowledge Discovery and Data Mining (KDD '09), June 28-July 01, 2009, ACM, Paris, France, ISBN:978-1-60558-495-9, pp: 467-476.

Lappas, T., K. Liu and E. Terzi, 2011. A Survey of Algorithms and Systems for Expert Location in Social Networks. In: Social Network Data Analytics, Aggarwal, C.C. (Ed.). Springer, Boston, Massachusetts, USA., ISBN:978-1-4419-8461-6, pp: 215-241.

Leicht, E.A., P. Holme and M.E.J. Newman, 2006. Vertex similarity in networks. Phys. Rev. E., 73: 1-10.

Leroy, V., B.B. Cambazoglu and F. Bonchi, 2010. Cold start link prediction. Proceedings of the 16th ACM SIGKDD International Conference on Knowledge Discovery and Data Mining (KDD '10), July 25-28, 2010, ACM, Washington, DC, USA., ISBN:978-1-4503-0055-1, pp: 393-402.

Leskovec, J., D. Huttenlocher and J. Kleinberg, 2010. Predicting positive and negative links in online social networks. Proceedings of the 19th International Conference on World Wide Web (WWW '10), April 26-30, 2010, ACM, Raleigh, North Carolina, USA., ISBN:978-1-60558-799-8, pp: 641-650.

Levien, R., 2004. Attack-resistant trust metrics. Ph.D Thesis, University of California at Berkeley, Berkeley, California, USA.

Liu, B., 2012. Sentiment Analysis and Opinion Mining, Synthesis Lectures on Human Language Technologies. Morgan \& Claypool Publishers, San Rafael, California, USA., ISBN:9781608458851, Pages: 167. 
Liu, B., 2015. Sentiment Analysis: Mining Opinions, Sentiments and Emotions. Cambridge University Press, Cambridge, UK., ISBN:978-1-107-01789-4, Pages: 366.

Liu, L., J. Tang, J. Han, M. Jiang and S. Yang, 2010. Mining topic-level influence in heterogeneous networks. Proceedings of the 19th ACM International Conference on Information and Knowledge Management (CIKM 10), October 26-30, 2010, ACM, Toronto, Canada, ISBN:978-1-4503-0099-5, pp: 199-208.

Lops, P., D.M. Gemmis and G. Semeraro, 2011. Content-Based Recommender Systems: State of the Art and Trends. In: Recommender Systems Handbook, Ricci, F., R. Lior, S. Bracha and B.K. Paul (Eds.). Springer, Berlin, Germany, ISBN:978-0-387-85819-7, pp: 73-105.

Meftahi, B. and O.B.B. Saidi, 2013. A new homogeneity inter-clusters measure in semisupervised clustering. Intl. J. Comput. Appl., 66: 37-45.

Nepal, S., W. Sherchan and C. Paris, 2011. Strust: A trust model for social networks. Proceedings of the 2011 IEEE 10th International Conference on Trust, Security and Privacy in Computing and Communications, November 16-18, 2011, IEEE, Changsha, China, ISBN:978-1-4577-2135-9, pp: 841-846.

Newman, M.E.J., 2001. Clustering and preferential attachment in growing networks. Phys. Rev. E., 64: $1-13$.

Newman, M.E.J., 2010. Networks: An Introduction. Oxford University Press, Oxford, UK., ISBN:9780191500701, Pages: 784.

Nguyen, N.P., T.N. Dinh, Y. Xuan and M.T. Thai, 2011. Adaptive algorithms for detecting community structure in dynamic social networks. Proceedings of the 2011 IEEE International Conference on INFOCOM, April 10-15, 2011, IEEE, Shanghai, China, ISBN:978-1-4244-9919-9, pp: 2282-2290.

O'Donovan, J. and B. Smyth, 2005. Trust no one: Evaluating trust-based filtering for recommenders. Proceedings of the 19th International Joint Conference on Artificial Intelligence (IJCAI'05), July 30-August 5, 2005, Edinburgh, Scotland, UK., pp: 1663-1665.

Palla, G., Derenyi, I., I. Farkas and T. Vicsek, 2005. Uncovering the overlapping community structure of complex networks in nature and society. Nature, 435 : 814-818.

Parimi, R. and D. Caragea, 2011. Predicting friendship links in social networks using a topic modeling approach. Proceedings of the 15th Pacific-Asia Conference on Knowledge Discovery and Data Mining (PAKDD 2011), May 24-27, 2011, Springer, Berlin, Germany, ISBN:978-3-642-20846-1, pp: 75-86.
Qi, G.J., C.C. Aggarwal and T.S. Huang, 2012. On clustering heterogeneous social media objects with outlier links. Proceedings of the 5th ACM International Conference on Web Search and Data Mining (WSDM '12), February 08-12, 2012, ACM, Seattle, Washington, USA., ISBN: 978-1-45030747-5, pp: 553-562.

Ravasz, E., A.L. Somera, D.A. Mongru, Z.N. Oltvai and A.L. Barabasi, 2002. Hierarchical organization of modularity in metabolic networks. Science, 297: 1551-1555.

Rawashdeh, M., H.N. Kim and A. El Saddik, 2013. Social media annotation and tagging based on folksonomy link prediction in a tripartite graph. Proceedings of the International Conference on Multimedia Modeling, January 7-9, 2013, Springer, Berlin, Germany, ISBN:978-3-642-35724-4, pp: 24-35.

Resnick, P., N. Iacovou, M. Suchak, P. Bergstrom and J. Riedl, 1994. GroupLens: An open architecture for collaborative filtering of netnews. Proceedings of the 1994 ACM Conference on Computer Supported Cooperative Work, October 22-26, 1994, ACM, Chapel Hill, North Carolina, USA, ISBN: 0-89791-689-1, pp: 175-186.

Ricci, F., L. Rokach, B. Shapira and P.B. Kantor, 2011. Recommender Systems Handbook. Springer, Berlin, Germany, ISBN:978-0-387-85820-3, Pages: 842.

Saito, K., R. Nakano and M. Kimura, 2008. Prediction of information diffusion probabilities for independent cascade model. Proceedings of the International Conference on Knowledge-Based and Intelligent Information and Engineering Systems, September 3-5, 2008, Springer, Berlin, Germany, ISBN:978-3-540-85566-8, pp: 67-75.

Schifanella, R., A. Barrat, C. Cattuto, B. Markines and F. Menczer, 2010. Folks in folksonomies: Social link prediction from shared metadata. Proceedings of the 3rd ACM International Conference on Web Search and Data Mining (WSDM '10), February 04-06, 2010, ACM, New York, USA., ISBN:978-1-60558-889-6, pp: 271-280.

Schubert, E., M. Weiler and H.P. Kriegel, 2014. Signitrend: Scalable detection of emerging topics in textual streams by hashed significance thresholds. Proceedings of the 20th ACM SIGKDD International Conference on Knowledge Discovery and Data Mining (KDD '14), August 24-27, 2014, ACM, New York, USA., ISBN:978-1-4503-2956-9, pp: 871-880.

Sellami, K., M. Ahmed-Nacer and P. Tiako, 2014. From social network to semantic social network in recommender system. Intl. J. Comput. Sci. Issues, 9: 501-507. 
Shamma, D.A., L. Kennedy and E.F. Churchill, 2011. Peaks and persistence: Modeling the shape of microblog conversations. Proceedings of the ACM 2011 International Conference on Computer Supported Cooperative Work (CSCW '11), March 19-23, 2011, ACM, Hangzhou, China, ISBN:978-1-4503-0556-3, pp: 355-358.

Sherchan, W., S. Nepal and C. Paris, 2013. A survey of trust in social networks. ACM. Comput. Surv. CSUR., 45: 1-33.

Singh, A., D. Bansal and S. Sofat, 2014. Privacy preserving techniques in social networks data publishing-a review. Intl. J. Comput. Appl., 87: 9-14.

Sulieman, D., M. Malek, H. Kadima and D. Laurent, 2012. Exploiting Semantic and Social Information in Recommendation Algorithms. In: Information Search, Integration and Personalization, Tanaka, Y., N. Spyratos, T. Yoshida and C. Meghini, (Eds.). Springer, Berlin, Germany, ISBN:978-3-64240139-8, pp: 91-100.

Sun, Y., J. Han, P. Zhao, Z. Yin, H. Cheng and T. Wu, 2009. Rankclus: Integrating clustering with ranking for heterogeneous information network analysis. Proceedings of the 12th International Conference on Extending Database Technology: Advances in Database Technology, March 23-26, 2009, New York, USA., pp: 565-576.

Symeonidis, P. and N. Mantas, 2013. Spectral clustering for link prediction in social networks with positive and negative links. Soc. Network nal. Min., 3: 1433-1447.

Tang, J., T. Lou and J. Kleinberg, 2012. Inferring social ties across heterogenous networks. Proceedings of the 5th ACM International Conference on Web Search and Data Mining (WSDM '12), February 08-12, 2012, ACM, Seattle, Washington, USA., ISBN:978-1-45030747-5, pp: 743-752.

Tang, L. and H. Liu, 2009. Scalable learning of collective behavior based on sparse social dimensions. Proceedings of the 18th ACM Conference on Information and Knowledge Management (CIKM '09), November 02-06, 2009, ACM, Hong Kong, China, ISBN:978-1-60558-512-3, pp: 1107-1116.

Tang, W., H. Zhuang and J. Tang, 2011. Learning to infer social ties in large networks. Proceedings of the Joint European Conference on Machine Learning and Knowledge Discovery in Databases, September 19-23, 2011, Springer, Berlin, Germany, ISBN:978-3-642-23807-9, pp: 381-397.
Weng, L., J. Ratkiewicz, N. Perra, B. Goncalves and C. Castillo et al., 2013. The role of information diffusion in the evolution of social networks. Proceedings of the 19th ACM SIGKDD International Conference on Knowledge Discovery and Data Mining (KDD '13), August 11-14, 2013, ACM, Chicago, Illinois, USA., ISBN:978-1-4503-2174-7, pp: 356-364.

Xia, Z. and Z. Bu, 2012. Community detection based on a semantic network. Knowl. Based Syst., 26: 30-39.

Xiang, R., J. Neville and M. Rogati, 2010. Modeling relationship strength in online social networks. Proceedings of the 19th International Conference on World Wide Web, April 26-30, 2010, ACM, Raleigh, North Carolina, ISBN:978-1-60558-799-8, pp: 981-990.

Xiang, R., J. Neville and M. Rogati, 2010. Modeling relationship strength in online social networks. Proceedings of the 19th International Conference on World Wide Web, April 26-30, 2010, ACM, Raleigh, North Carolina, ISBN:978-1-60558-799-8, pp: 981-990.

Xie, S., 2014. A collaborative filtering recommendation algorithm improved by trustworthiness. Intl. J. Future Gener. Commun. Networking, 7: 35-46.

Yang, J. and J. Leskovec, 2010. Modeling information diffusion in implicit networks. Proceedings of the 2010 IEEE International Conference on Data Mining, December 13-17, 2010, IEEE, Sydney, Australia, ISBN:978-1-4244-9131-5, pp: 599-608.

Yang, J. and S. Counts, 2010. Predicting the speed, scale and range of information diffusion in twitter. Proceedings of the 4th International AAAI Conference on Weblogs and Social Media, May 23-26, 2010, Association for the Advancement of Artificial Intelligence, Menlo Park, California, USA., pp: 335-358.

Yoshida, T., 2012. Line Graph for Weighted Networks toward Overlapping Community Discovery. Intelligent Decision Technologies, Watada, J., T. Watanabe, G. Phillips-Wren, R. Howlett and L. Jain (Eds.). Springer, Berlin, Germany, ISBN:978-3-64229919-3, pp: 403-413.

Zhang, J., J. Tang and J. Li, 2007. Expert finding in a social network. Proceedings of the 12th International Conference on Database Systems for Advanced Applications (DASFAA 2007), April 9-12, 2007, Springer, Bangkok, Thailand, pp:1066-1069.

Zheng, X., Z. Zeng, Z. Chen, Y. Yu and C. Rong, 2015. Detecting spammers on social networks. Neurocomputing, 159: 27-34.

Zhou, T., L. Lu and Y.C. Zhang, 2009. Predicting missing links via local information. Eur. Phys. J. B., 71: 623-630. 
Zhou, Y. and L. Liu, 2013. Social influence based clustering of heterogeneous information networks. Proceedings of the 19th ACM SIGKDD International Conference on Knowledge Discovery and Data Mining (KDD '13), August 11-14, 2013, ACM, Chicago, Illinois, USA., ISBN:978-1-4503-2174-7, pp: 338-346.

Zhou, Y., H. Cheng and J.X. Yu, 2009. Graph clustering based on structural/attribute similarities. Proc. VLDB Endowment, 2: 718-729.

Zhou, Y., H. Cheng and J.X. Yu, 2010. Clustering large attributed graphs: An efficient incremental approach. Proceedings of the 2010 IEEE International Conference on Data Mining, December 13-17, 2010, IEEE, Sydney, Australia, ISBN:978-1-4244-9131-5, pp: 689-698.
Ziegler, C.N. and G. Lausen, 2004. Spreading activation models for trust propagation. Proceedings of the IEEE International Conference on E-Technology, E-Commerce and E-Service (EEE'04), March 28-31, 2004, IEEE, Taipei, Taiwan, ISBN:0-7695-2073-1, pp: 83-97.

Ziegler, C.N. and G. Lausen, 2005. Propagation models for trust and distrust in social networks. Inf. Syst. Front., 7: 337-358.

Ziegler, C.N. and J. Golbeck, 2007. Investigating interactions of trust and interest similarity. Decis. Support Syst., 43: 460-475. 